confirm that it is realistic and assess whether PR benefits can be sustained.

Methods Three suitable venues, geographically distributed to maximise access, and two exercise instructors (completed Loughborough Training for Chronic Respiratory Patients) were identified. Patients completing PR were recruited into $1 \times$ weekly LTE groups. Outcomes (demographic, disease severity, functional capacity (6 min walk test (6MWT), emotional (Hospital Anxiety and Depression HAD score, CAT and Chronic Respiratory Questionnaire CRQ)) were collected at baseline, 6 and 12 months. Patient and carer satisfaction was recorded at 6 and 12 months. Attendance data were monitored throughout. Hospital admissions for $12 / 12$ before and after LTE commenced, continues to be audited.

Results 60 COPD patients (Mean (SD) age 68.75 (10.31) yrs, FEV 1.27 (0.56) 1, 43\% male, 22\% current smokers, three on LTOT) were referred for LTE June 2010-May 2011. 20/60 (33\%) never attended, 14/60 (23\%) dropped out within $2(1-7)$ months (median (range). 26/60 (43\%) continue to exercise, median (range) since starting 5 (1-12) months with 50 (28)\%. (Mean (SD) classes attendance. CAT, CRQ, HADS and 6MWT findings are given in the Abstract P149 table 1. Satisfaction surveys indicate high levels of satisfaction with venue, instructor and content. No adverse events occurred during the classes. Direct cost per class (hall hire and instructor) $£ 63$.

Abstract P149 Table 1 Health related quality of life and exercise capacity at end-PR, 6 months and 12 months of long-term exercise

\begin{tabular}{lccc}
\hline & $\begin{array}{l}\text { Baseline, } \mathbf{n}=\mathbf{6 0}, \\
\text { mean (SD) }\end{array}$ & $\begin{array}{l}\mathbf{6} \text { Months, } \mathbf{n = 1 1 ,} \\
\text { mean (SD) }\end{array}$ & $\begin{array}{l}\text { 12 Months, } \mathbf{n}=\mathbf{3}, \\
\text { mean (SD) }\end{array}$ \\
\hline MRC & $3.2(0.8)$ & & \\
CAT score & $22.2(6.8)$ & $12.4(7.4)$ & $17.0(14.1)$ \\
CR0 Dyspnoea & $3.3(1.4)$ & $4.2(1.6)$ & $4.9(1.9)$ \\
CR0 fatigue & $4.1(1.5)$ & $4.9(1.2)$ & $4.7(2.5)$ \\
CR0 emotional function & $4.8(1.6)$ & $5.3(1.3)$ & $4.8(2.9)$ \\
CR0 mastery & $4.9(1.7)$ & $5.8(1.4)$ & $4.1(1.9)$ \\
HADS anxiety & $7.4(5.1)$ & $4.3(3.3)$ & $6.0(6.0)$ \\
HADS depression & $5.2(4.2)$ & $3.6(2.5)$ & $4.7(4.7)$ \\
6 MWT (metres) & $353(116)$ & $338(83)$ & $330(122)$ \\
\hline
\end{tabular}

Conclusions This pilot demonstrates feasibility of providing community-based LTE groups using instructors trained to exercise people with COPD. Patients who choose to attend LTE have moderate COPD, but high CAT scores indicating important disease impact on daily functioning. Attendance rate was high for a patient group susceptible to exacerbations, possibly reflecting high patient satisfaction. Preliminary findings suggest LTE in group settings promotes maintenance of benefits acquired from PR with further improvement in health-related quality of life (CRO and CAT).

\section{P150 USE OF AMBULATORY OXYGEN THERAPY IN PULMONARY REHABILITATION}

doi:10.1136/thoraxjnl-2011-201054c.150

D Anderson, S Ferrer Valls, S Church, S Agarwal. St Helens and Knowsley teaching Hospitals NHS Trust, Prescot, UK

Introduction and Objectives Pulmonary rehabilitation (PR) is proven to improve oxygen exercise capacity, health status and reduce dyspnoea in patients diagnosed with Chronic Obstructive Pulmonary Disease. Previous studies have shown no significant difference in rehabilitation outcomes following use of supplemental oxygen during pulmonary rehabilitation. Unfortunately, a few patients are unable to exercise due to hypoxia related dyspnoea depriving them of the benefit of PR. We hypothesised that the use of Ambulatory Oxygen Therapy (AOT) in patients with exercise induced desaturation would increase their capability of undergoing PR and have an overall positive outcome.

Methods Requirement of AOT is routinely assessed at our trust prior to commencing PR. Incremental oxygen is used to decide on the flow rate needed during PR. A retrospective study was performed to evaluate the benefits of AOT in patients desaturating on exertion. Data were analysed using Excel.

Results 218 patients were enrolled for the programme from January 2009 to July 2010, 46 excluded as not suitable or did not attend. Numbers studied-172: 59\% male, mean age 70 (range 45-90), 25\% current smokers. On initial endurance exercise test $27 \%(n=47)$ were noted to have exercise induced desaturation. Seven patients were already on long-term oxygen therapy. Median prescribed flow rate was $21 /$ min during PR. The mean distance walked on initial endurance test was $150 \mathrm{~m}$. This improved significantly to $263 \mathrm{~m}$ $(p<0001)$ with AOT. $68 \%(n=32)$ attended for post PR endurance exercise testing, $25 \%$ no longer required AOT (did not desaturate). Of the 22 patients (46\%) who continued to require AOT, 14 were prescribed. Following 2 months of the prescription, AOT was withdrawn in four patients as they found the cylinder too heavy or did not benefit.

Conclusions AOT has shown significant improvement in rehabilitation outcomes in patients with exercise induced desaturation. A quarter of these patients did not require any further ambulatory oxygen therapy following pulmonary rehabilitation.

Abstract P150 Table 1

\begin{tabular}{lll}
\hline Paired t test & $\begin{array}{l}\text { Mean distance on initial } \\
\text { endurance exercise test, 150 m }\end{array}$ & p Values \\
\hline Mean distance on AOT & $263 \mathrm{~m}$ & 0.0001 \\
Mean distance post PR & $277 \mathrm{~m}$ & 0.0069 \\
Mean distance post PR + AOT & $356 \mathrm{~m}$ & 0.0003 \\
\hline
\end{tabular}

\section{P151 COST OF PULMONARY REHABILITATION IS OFFSET BY REDUCTION IN HEALTHCARE UTILISATION}

doi:10.1136/thoraxjnl-2011-201054c.151

${ }^{1} \mathrm{~S}$ Kibe, ${ }^{1} \mathrm{D}$ Frost, ${ }^{2} \mathrm{~S}$ Hart. ${ }^{1}$ Scarborough General Hospital, Scarborough, UK; ${ }^{2}$ Castle Hill Hospital, Hull, UK

151 patients completed pulmonary rehabilitation over a period of over 4 years at our hospital. Retrospective analysis of 71 patients from this group was done. This showed that there was a significant difference between pre and post rehabilitation in FVC, VC, HADS depression score \& SWT (shuttle walk test distance). Analysis of a sub group of 37 patients at 6 months after pulmonary rehabilitation showed that there were still significant differences in HADS depression \& SWT, but not the other variables. We were able to establish a significant reduction in healthcare utilisation, both in hospital admissions and in out patient attendances. This was independent of the reported level of benefit on CRDQ scores. "Responders" (patients with a more than 1.5 increase in each of their CRDO scores) had similar reduction in healthcare utilisation compared to "non-responders" (patients with $<0.5$ difference or deterioration in their CRDQ scores). At our hospital the approximate cost of pulmonary rehabilitation is 375 pounds per person. Out patient (respiratory) appointments and hospital admissions 